\title{
A simple procedure to tailor the compositional gradient of copolymeric materials
}

\author{
Helmut Reinecke, ${ }^{1}$ María Rosa Aguilar, ${ }^{1}$ Güldem Güven, ${ }^{2}$ Erhan Piskin, ${ }^{2}{ }^{3}$ Alberto \\ Gallardo ${ }^{1^{*}}$ \\ ${ }^{1}$ Institute of Polymer Science and Technology, CSIC. Juan de la Cierva 3, 28006 \\ Madrid, Spain; tel: -34-95622900; fax: 915644853. e-mail: icthr14@ictp.csic.es, \\ mraguilar@ictp.csic.es and gallardo@ictp.csic.es \\ ${ }^{2}$ Hacettepe University, Chem. Eng. Dept. and Bioeng. Div., Beytepe 06800 Ankara, \\ Turkey; fax: 0312 4406214; e-mail: gguven@hacettepe.edu.tr \\ ${ }^{3}$ BIYOMEDTEK: Center for Biomedical Technologies, Perisuyu Sok. No:7, \\ Beysukent, 06800 Ankara, Turkey; fax: 031223636 57; email: erhanpiskin@ \\ biyomedtek.com
}

(Received: 16 January, 2008; published: 17 February, 2009)

\begin{abstract}
Copolymeric structures with compositional gradient were produced by "in situ" radical polymerization -in water and with redox initiation- of acrylic acid (AA) and 2-hydroxyethyl methacrylate (HEMA) in the presence of an inhibitor (hydroquinone). The inhibitor has a key role in controlling the mixing of precursor solutions because it produces a retardation of the polymerization. Raman spectral analysis confirmed the changes in the composition along the long axis of the produced cylindrical materials. Compression tests were also performed, exhibiting the gradient and reference material's unique properties, which were well correlated to the compositional pattern.
\end{abstract}

\section{Introduction}

Gradient materials exhibit a continuous change in composition of components from one side of the material to the other. Initially they have been developed for metals and ceramics, and are currently receiving increasing attention in the field of polymer science. Different gradients of additives, fillers, etc.[1-3] have been described, some of them obtained by "in situ" polymerization, as the dyed gradients reported by Masere et al.[2] and Chekanov et al.[3] produced using isothermal frontal polymerization. In addition to this, most of the studies have mainly focused on the preparation of gradients of blends [4-6] starting from the parent polymer components. Some of these gradients have been also prepared by "in situ" polymerization including gradient IPN or semilPN gradients [7]. Duijnhoven and Bastiaansen have introduced an interesting approach in which centrifugal forces have been applied to generate compositional gradients in polymer/monomer mixtures which have been fixed then by polymerization [8].

In this study we propose a very simple technique to produce compositional copolymer gradients. Two well known hydrophilic comonomers, namely acrylic acid (AA) and 2-hydroxyethyl methacrylate (HEMA) have been "in situ" polymerized by radical polymerization by using a mixture of two water soluble initiators with or without using an inhibitor. This paper shows the formation of the gradient materials 
and reports the role of the inhibitor controlling the changes in the composition and mechanical properties (compression strength) in gradient structures.

As it has been described in the next sections, the procedure we describe here is based on this: we allow parent comonomer solutions to mix until a point where the gradient monomer composition is fixed by polymerization or curing. The latent period controls the gradient and is defined in here by the percentage of inhibitor in a radical redox initiation.

\section{Results and discussion}

Representative optical photographs of the cylinders, one from the "-H series" (without inhibitor) and from the " $+\mathrm{H}$ series" (with inhibitor) are given in Figure 1, together with the Raman analysis -that will be discussed later-. The differences between these two copolymeric materials can be clearly seen in these photographs, even with naked eye. A very sharp transition is observed in the "-H cylinder" in which the transparent part is mainly composed of $A A$, while the upper opaque part contains more HEMA. In the case of "+H cylinder" a gradual change in transparency (colour) is observed.

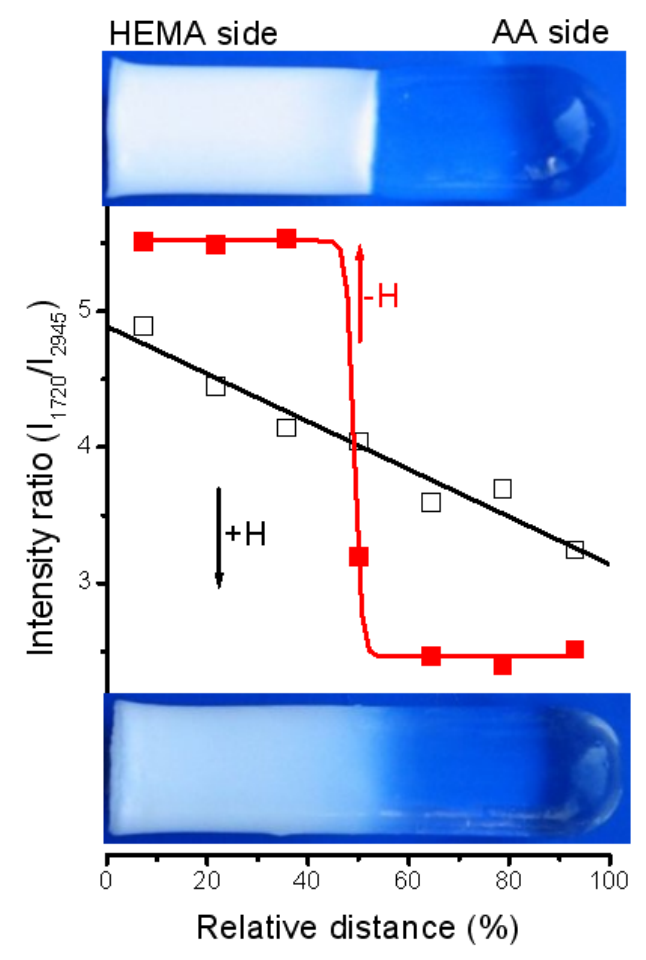

Fig. 1. Representative optical photographs of cylinders: (up) "-H series" (without inhibitor) and (down) "+H series" (with inhibitor), together with the changes in the intensity ratio $\left(\mathrm{I}_{1720} / \mathrm{I}_{2945}\right)$ along the long axis of the cylinders: For "-H series" (filled square) and for "+H series" (hollow square).

Figure 2 describes schematically the polymerization in both the " $-\mathrm{H}$ series" and " $+\mathrm{H}$ series". Note that all parameters in both groups were identical except that we have used more initiators and plus extra inhibitor in order to reach similar polymerization rates. In the "-H series", polymerizations in both parts (the upper and lower) started 
immediately, allowing the monomer diffusions (mixing) only in the neighbours of the initial interface. Therefore, a two-component material (it may be considered also as a sharp gradient) was observed as shown also in Figure 1 (up). However, in the reaction of the $+\mathrm{H}$ series, polymerization started 15 min after the Solution III was poured on to the Solution IV, and during this latency period monomers were able to diffuse in the neighbouring solution and get mixed. As a consequence a smooth gradient was observed in this case (see also Figure 1-down).

A $-\mathrm{H}$, without inhibitor

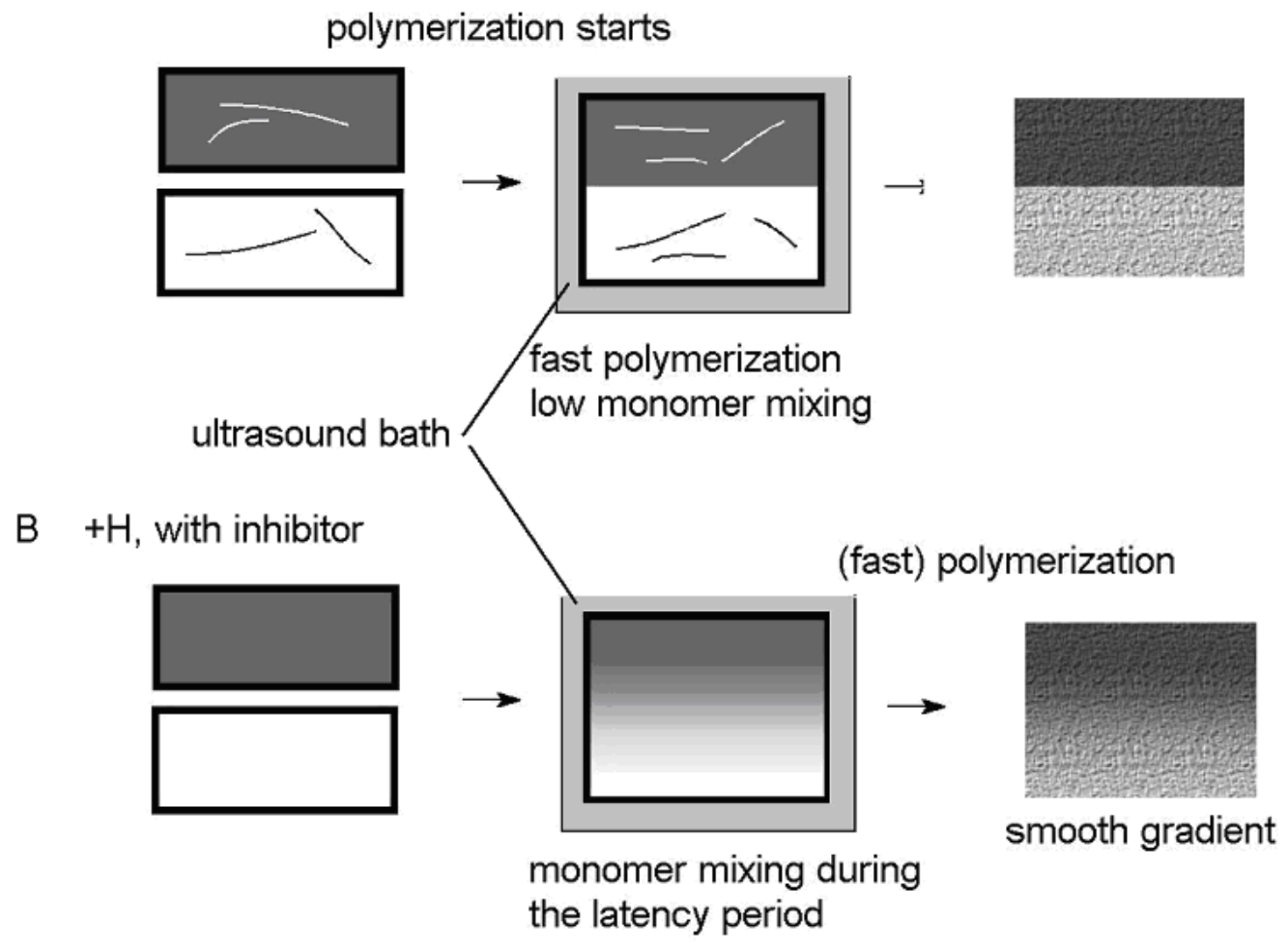

$\square$ solution of monomer 1

Fig. 2. Schematic representation of production of gradient copolymeric materials: (A) "-H series" (without inhibitor) and (B) "+H series" (with inhibitor).

In order to confirm the structures of the cylindrical materials produced in the both " $-\mathrm{H}$ series" and "+H series" we have used a Raman microscopy technique, in which the ratio of intensities at $1720 \mathrm{~cm}^{-1}$ ( $\left(_{1720}\right.$, corresponding to the carbonyl band) and at $2945 \mathrm{~cm}^{-1}\left(\mathrm{I}_{2945}\right.$, corresponding to the alkyl band) were used to present the changes in the relative amounts of AA and HEMA along the long axis of the cylinders. Figure 1 gives the changes of the intensity ratio $\left(I_{1720} / I_{2945}\right)$ with the long axis. Note that these ratios were 5.5 and 2.5 for PHEMA and PAA homopolymers, respectively. The values for copolymers of HEMA and AA were in these limits. Figure 1 clearly demonstrates that in absence of the inhibitor (the "-H series"), two materials 
consisting of either practically pure PAA homopolymer (right side) or pure PHEMA homopolymer (left side) are formed and connected to each other with a sharp transition almost in the middle of the cylinder. On the other hand, in the system where the inhibitor has been added to retard the polymerization (the "+H series"), both monomer solutions had enough time to mix and form a copolymeric material with a nearly linear concentration gradient of AA or HEMA leading to a smooth type of gradient material.

Gradient materials are materials with unique properties due to the particular molecular order along a spatial dimension. In this respect, the two series of cylinders prepared in this study exhibited different longitudinal compressibilities, as is shown in Figure 3.

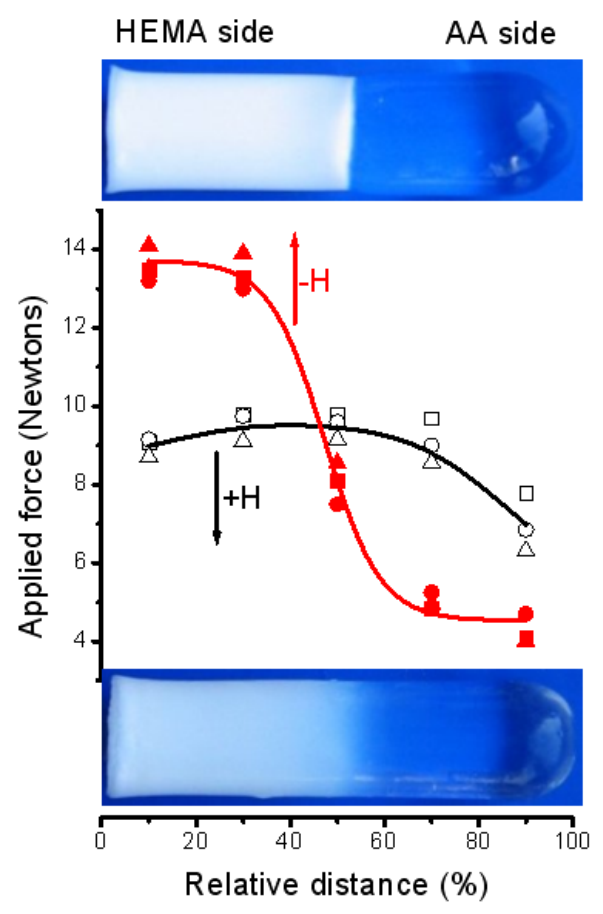

Fig. 3. Changes in the compression forces along the long axis of the cylinders: For "-H series" (filled square) and for "+H series" (hollow square).

Note that compression was applied along the longitudinal dimension of the cylinders and the applied force was measured at constant strain, as described in the experimental part. The average values and standard deviation of the values obtained in the parallel studies in which three cylinders from each group were tested, are given in the Figure. Both cylinders exhibited elastomeric behaviour under the compressional force applied. In the "-H series", there was a very significant difference in the level of applied pressure measured in the two regions, parallel to the compositional changes exhibited in Figure 1. Higher strengths were observed with less swellable PHEMA homopolymer part (around 13-14 Newton), while the level of applied forces were about 4-5 Newton in the PAA part. The force applied in the middle of the cylinder is around the average of both sides. However, the situation was quite different for the cylinders obtained in the the "+H series". The change of the applied force was not that significant along the long axis of the cylinder; it was around 
8-9 Newton in most of the regions, and decreased about to 6-7 Newton for the AA end (at the bottom of the reaction tube), which is also in agreement with the better monomer mixing provided by the retardation of the inhibitor. In this case the response was not linear because the mechanical performance depends not only on the composition but also on the microstructure and on the inter- and intramolecular interactions. The decrease of mechanical property in the HEMA side may be attributed to an edge effect. These results demonstrate that the mechanical performance -and the functionality- is defined by the type of structure.

In conclusion, we proposed that gradient material with tailored structures could be produced by controlling the rate and fate of polymerization, i.e., by simply selecting the correct monomers with correct initiators and inhibitors.

\section{Experimental part}

\section{Materials}

HEMA (Fluka) and AA (Merck) were purified by distillation at reduced pressure. Ethylene glycol dimethacrylate (EGDMA, the cross-linker from Fluka), hydroquinone (HQ, "inhibitor" from Aldrich), ammonium persulfate (APS, the "initiator 1" from Aldrich) and ascorbic acid (vitamine C, VC, the "initiator 2" from Aldrich) were used as received. The phosphate buffer solution ( $\mathrm{pH}$ 7.4) was prepared by dissolving commercial tablets (Sigma) in distilled water.

\section{Polymerization}

Solutions of both initiators, APS and VC, were prepared as follows: $0.5 \mathrm{~g}$ of each initiator was dissolved in $5 \mathrm{ml}$ of buffer $\mathrm{pH} \mathrm{7.4}$. The solution of inhibitor was prepared freshly by dissolving $0.2 \mathrm{~g}$ of hydroquinone in $0.5 \mathrm{ml}$ of buffer. Two series of studies were conducted, i.e., without using inhibitor (called as "-H series" in this text) and with inhibitor (called as "+H series"). The recipes are given in Table 1.

Tab. 1. Recipes for the polymerizations.

\begin{tabular}{cccccccc}
\hline & $\begin{array}{c}\text { HEMA } \\
(\mathrm{ml})\end{array}$ & $\begin{array}{c}\text { AA } \\
(\mathrm{ml})\end{array}$ & $\begin{array}{c}\text { Buffer } \\
(\mathrm{ml})\end{array}$ & $\begin{array}{c}\text { EGDMA } \\
(\mathrm{ml})\end{array}$ & $\begin{array}{c}\text { Initiator } \\
1(\mathrm{ml})\end{array}$ & $\begin{array}{c}\text { Initiator } \\
2(\mathrm{ml})\end{array}$ & $\begin{array}{c}\text { Inhibitor } \\
(\mathrm{ml})\end{array}$ \\
\hline For "-H series" & & & & & & & \\
$\quad$ Solution I & 1 & - & 1.1 & 0.05 & 0.15 & 0.15 & - \\
$\quad$ Solution II & - & 0.75 & 1.1 & 0.05 & 0.15 & 0.15 & - \\
For "+H series" & & & & & & & \\
$\quad$ Solution III & 1 & - & 0.75 & 0.05 & 0.25 & 0.25 & 0.15 \\
$\quad$ Solution IV & - & 0.75 & 0.75 & 0.05 & 0.25 & 0.25 & 0.15 \\
\hline
\end{tabular}

The gradient materials were obtained at room temperature as follows: The Solutions I and II in the "-H series" and III and IV in the "+H series" were freshly prepared. The AA solution (II or IV) was poured in a glassy cylindrical test tube (diameter $1 \mathrm{~cm}$, length $6 \mathrm{~cm}$ ). The HEMA solution (I or III) was then deposited with a Pasteur pipette over the AA solution and the test tube was immediately placed in an ultrasonic bath for 60 minutes at room temperature. After this time, the glass tube was broken and the cylinders were immersed in distilled water in order to remove residuals, e.g. monomer, initiator and inhibitor by extraction. The medium was replenished with pure 
water three times in 24 hours and the cylinders were kept in water until use. Three cylinders of each type were prepared.

\section{Raman Spectral Microscopy}

The compositions of the materials were analysed using Raman microscopy. Raman spectra of different sites of the long axis of the cylinders were recorded on a Renishaw Ramascope spectrometer with the 632.8-nm line of a He-Ne laser. The instrument is equipped with a Peltier cooled charge-coupled device (CCD) detector, a holografic grating (1800 grooves/mm), and two Raman holografic edge filters, which prevent the backscattered laser radiation from entering the spectrograph. The stigmatic single spectrograph is attached to an Olympus $\mathrm{BH} 2$ microscope.

\section{Compression Tests}

Compression tests were carried out in a Shimadzu Autograph AGS-J, and using an adapter $-1 \mathrm{~cm}$ diameter- to test the cylinders in different positions along the longitudinal dimension, perpendicularly to the long axis of the cylinder. Force applied at constant strain of $3 \mathrm{~mm}$ was measured, using a crosshead speed range of 1 $\mathrm{mm} / \mathrm{min}$.

\section{Acknowledgements}

Study was supported by grant MAT 2007-63355 from the spanish CICYT and from the EXPERTISSUES Network of Excellence (NoE). Authors thank Prof. Dr. Riza Gürbüz and Gül Çevik -from the Metallurgical \& Materials Engineering, M.E.T.U. Ankara, Turkey- for the compression measurements. David Gómez from Polymer Institute in Madrid is also acknowledged for the SEM measurements. A.G. acknowledges the "Secretaria de Estado de Universidades e Investigación" of the Spanish Ministry of Education and Science for the concession of a mobility grant.

\section{References}

[1] Lipatov, Y.S.; Karabanova, L.V. J. Mater. Sci. 1995, 30, 2475.

[2] Masere, J.; Lewis, L.L.; Pojman, J.A. J. Applied Polym. Sci. 2001, 80, 686.

[3] Chekanov, Y.A.; Pojman, J.A. J. Applied Polym. Sci. 2000, 78, 2398.

[4] Zhao L, Tsuchiya K, Inoue Y. Macromol. Biosci. 2004, 4, 699.

[5] Gallant FM, Bruck HA, Kota A J. Compos. Mater. 2004, 38, 1873.

[6] Hexig B, Alata H, Asakawa N, Inoue Y. Adv. Funct. Mater. 2005, 15, 1630.

[7] Karabanova, L.V.; Mikhalovsky, S.V.; Lloyd, A.W.; Boiteux, G.; Sergeeva, L.M.; Novikova, T.I.; Lutsyk, E.D.; Meikle S. J. Mater. Chem. 2005, 15, 499.

[8] Duijnhoven, F.; Bastiaansen, C. Adv. Mater. 1999, 11, 567. 